IFT-99/068

$\mathrm{PM} / 99-34$

SLAC-PUB-8249

\title{
Supersymmetric Higgs Boson Pair Production: Discovery Prospects at Hadron Colliders
}

\author{
A. Belyaev ${ }^{1,2}$, M. Drees ${ }^{1,3}$, J. K. Mizukoshi ${ }^{4}$ \\ ${ }^{1}$ Instituto de Física Teórica, Universidade Estadual Paulista, \\ Rua Pamplona 145, 01405-900, São Paulo, Brazil. \\ 2 Skobeltsyn Institute for Nuclear Physics, Moscow State University, 119 899, Moscow, Russia \\ ${ }^{3}$ Lab de Physique Mathematique, Univ. Montpellier II, Montpellier, France \\ ${ }^{4}$ Stanford Linear Accelerator Center, University of Stanford, \\ Stanford, CA 94309, USA
}

(August 15, 2018)

\begin{abstract}
We study the potential of hadron colliders in the search for the pair production of neutral Higgs bosons in the framework of the Minimal Supersymmetric Standard Model. We perform a detailed signal and background analysis, working out efficient kinematical cuts for the extraction of the signal. The important role of squark loop contributions to the signal is re-emphasized. If the signal is sufficiently enhanced by these contributions, it could even be observable at the next run of the upgraded Tevatron collider in the near future. At the LHC the pair production of light and heavy Higgs bosons might be detectable simultaneously.
\end{abstract}

14.80.Cp, 14.80.Ly

Typeset using REVTEX 


\section{INTRODUCTION}

The search for Higgs bosons is one of the most important tasks for experiments at present and future high energy colliders [1]. At $e^{+} e^{-}$colliders, precision measurements of many of their properties are in principle quite straightforward, given sufficient energy and luminosity [2]. However, the LEP collider is nearing the end of its lifetime without having detected any signal for Higgs bosons. Various plans for the construction of future (linear) $e^{+} e^{-}$ colliders exist, but the prospects for their realization remain uncertain. On the other hand, the Tevatron will soon start its next collider run with slightly increased beam energy and greatly increased luminosity; a few years later experiments at the LHC will commence taking data. It is therefore imperative to fully exploit the opportunities for Higgs boson searches at hadron colliders.

Unfortunately most signals for the production of neutral Higgs bosons at hadron colliders suffer from a low signal to background ratio, and/or use decay modes with very low branching ratios. Examples for the former case are associated $W H$ or $Z H$ production followed by $H \rightarrow b \bar{b}$ decays [3], while an example for the latter situation is single Higgs boson production followed by $H \rightarrow Z Z^{*} \rightarrow 4 \ell$ decays, if $m_{H}<2 M_{Z}$. The discovery of Higgs bosons at hadron colliders is therefore not straightforward; this is certainly true for the Tevatron, but also holds for the LHC, with the possible exception of one or two "gold-plated" modes (e.g., $W H$ [4] or $t \bar{t} H$ [5] production, followed by $H \rightarrow \gamma \gamma$ decays, leading to $\ell \nu \gamma \gamma X$ final states) which however have small cross sections. The study of their properties at hadron colliders is correspondingly even more difficult. It is therefore important to search for Higgs bosons in as many different final states as possible. On the one hand multiple positive signals will increase confidence that a Higgs boson has indeed been found, since different final states will have different systematic uncertainties, a major concern for final states with low signal to background ratio. Moreover, by comparing the strengths of (or bounds on) several signals, much can be learned about the couplings of the Higgs bosons.

In this paper we study the production of two neutral Higgs bosons in gluon fusion, followed by the decays of both bosons into $b \bar{b}$ pairs. We focus on the final states where both Higgs bosons have (nearly) the same mass, since the resulting kinematical constraint helps to reduce the background. The SM cross section for this process has been computed some time ago [6]; it was found to be too small to be useful. However, the scalar sector of the SM suffers from well-known naturalness problems. These can be cured by introducing Supersymmetry. Here we concentrate on the simplest potentially realistic supersymmetric model, the minimal supersymmetric standard model (MSSM) [7]. Several effects can greatly enhance the Higgs pair production cross section in the MSSM as compared to the SM:

- The MSSM contains two Higgs doublet superfields, and correspondingly two independent vacuum expectation values (vevs). While the sum of the square of these vevs is fixed by the well-known masses of the $W$ and $Z$ bosons, their ratio, denoted by $\tan \beta$, is a free parameter of the MSSM. If $\tan \beta \gg 1$, the Yukawa coupling of the $b$-quark is enhanced by a factor $\sim \tan \beta$ compared to its SM value. It thus becomes comparable to the top quark Yukawa coupling for $\tan \beta \sim m_{t}\left(m_{t}\right) / m_{b}\left(m_{t}\right) \simeq 60$, which is possible in most realizations of the MSSM. For Higgs boson masses around $100 \mathrm{GeV}$ the squared $b$-loop contribution then exceeds the $t$-loop contribution, which is suppressed by the large mass of the top quark, by a factor $\sim 15.9$. 
- The MSSM contains three physical neutral Higgs bosons. If CP is conserved in the scalar sector of the theory, these states can be classified as two CP-even scalars $h, H$ (with $m_{h}<m_{H}$ ) and one $\mathrm{CP}$-odd pseudoscalar $A$. For some region of parameter space $\left(m_{A} \sim 300 \mathrm{GeV}, \tan \beta \lesssim 4\right)$ the branching ratio for $H \rightarrow h h$ decays is sizable. $h$ pair production through resonant $H$ exchange is then enhanced by a factor $\left(g M_{W} / \lambda_{t} \Gamma_{H}\right)^{2} \sim$ 100, where the Hhh coupling is $\mathcal{O}\left(g M_{W}\right), g$ being the $S U(2)$ gauge coupling, and $\lambda_{t}$ is the top Yukawa coupling [8.9].

- In addition to mandating the existence of (at least) two Higgs doublets, Supersymmetry also requires the presence of superpartners of all known quarks and leptons. This gives rise to new squark loop contributions to Higgs boson pair production through gluon fusion. In particular, contributions from loops involving $\tilde{b}$ or $\tilde{t}$ squarks can exceed those from $b$ and $t$ quark loops by more than two orders of magnitude [10]. This enhancement can occur for all values of $m_{A}$ and $\tan \beta$, but requires a fairly light squark mass eigenstate $\left(\tilde{t}_{1}\right.$ or $\left.\tilde{b}_{1}\right)$, as well as large trilinear Higgs-squark-squark couplings.

In this paper we show that neutral Higgs boson pair production can indeed be discovered in the $4 b$ final state at the LHC, if at least one of these possible enhancement factors is large. Under favorable circumstances, even the next run of the Tevatron collider could yield a signal. These results are based on a complete Monte Carlo simulation of signal and background, including realistic $b$-tagging efficiencies, parton showering and hadronization, and a simple parameterization of resolution smearing. The statistically most significant signal almost always comes from the $h h$ final state, in some cases augmented by $h A$ and $A A$ final states (for $m_{A} \simeq m_{h}$ ), but $H H$ production can also give a detectable signal. The $4 b$-jet signature for MSSM Higgs bosons has first been studied in refs. [8], with emphasis on $(h, H, A) b \bar{b}$ as well as $H \rightarrow h h, A A \rightarrow 4 b$ production. These earlier references did not study Higgs pair production in the continuum, however.

The remainder of this article is organized as follows. In Sec. II we describe our Monte Carlo simulation. Sec. III deals with the calculation of signal and background, and discusses the cuts used to maximize the statistical significance of the signal. These results are used in Sec. IV to estimate the discovery reach of the Tevatron and the LHC, and Sec. V contains our conclusions.

\section{MONTE CARLO SIMULATION}

In order to study the observability of the signal for Higgs pair production in the $4 b$ final state, we have written MC generators for complete sets of signal as well as background processes. These generators were designed as new external user defined processes for the PYTHIA 5.7/JETSET 7.4 package [11] using the standard PYTHIA routine PYUPEV.

A total of 6 processes contribute to the signal $(h h, h H, h A, H H, H A$ and $A A$ production). FORTRAN codes of the corresponding squared matrix elements, including squark loop contributions to $h h, h H, H H$ and $A A$ production, had already been available [10].

We used the CompHEP package [12 to generate background events on the parton level. This is based on a calculation of exact tree-level matrix elements for $g g, q \bar{q} \rightarrow b \bar{b} b \bar{b}$, as well as $g g, q \bar{q} \rightarrow Z b \bar{b}$ followed by $Z \rightarrow b \bar{b}$ decays. 
For both signal and background, the effects of initial and final state radiation, hadronization, as well as decay of the $b$-flavored hadrons have been taken into account. For simplicity we used the independent fragmentation scheme. CTEQ4L parton distributions [13 have been used everywhere. Since our matrix element calculation only includes the lowest nontrivial order in QCD perturbation theory, there is a considerable scale dependence. We chose the same value $Q$ for factorization and renormalization scales, including the scale of the running Yukawa couplings. For Higgs pair production this scale $Q$ was set to the average mass of the Higgs bosons in the final state, while for the $Z b \bar{b}$ background $Q=M_{Z}$ has been taken. Finally, when calculating the $4 b$ pure QCD background, we took $Q$ to be the average $b \bar{b}$ pair invariant mass. This choice of a rather high scale $Q$ should be conservative. While the predictions for both signal $S$ and background $B$ would be higher for smaller values of $Q$, leaving the ratio $S / B$ more or less the same, the significance $S / \sqrt{B}$ increases with decreasing $Q$.

PYTHIA demands that the scales for initial and final state radiation off user defined processes should be set explicitly by the user. These scales were chosen equal to factorization and renormalization scales.

In our analysis we used the cone algorithm for jet reconstruction, with cone size $\Delta R=$ $\sqrt{\Delta \varphi^{2}+\Delta \eta^{2}}=0.5$. The minimum $E_{T}$ threshold for a cell to be considered as a jet initiator has been chosen as $3 \mathrm{GeV}$, while the minimal summed $E_{T}$ for a collection of cells to be accepted as a jet has been set at $7 \mathrm{GeV}$; the cell size $\delta \eta \times \delta \phi$ has been taken as 8/25 $\times \pi / 12$. Finally, the energy of each jet was smeared, with resolution $\Delta E / E=0.8 / \sqrt{E}(E$ in $\mathrm{GeV})$ for the Tevatron and $\Delta E / E=0.5 / \sqrt{E}(E$ in $\mathrm{GeV})$ for the LHC. The mentioned resolutions are in agreement with those used in the Supersymmetry/Higgs RUN II workshop [17] and ATLAS/CMS studies of high $p_{T} b$-jets (see, for example, 18]), respectively.

Finally, we mention that the use of on-shell quark masses has been advocated when calculating the Yukawa couplings [9], based on experience with two-loop corrections to quark loop contributions to single Higgs production cross sections [14. This would increase the signal cross section in the region of large $\tan \beta$, where the dominant contributions involve the bottom Yukawa coupling, by more than a factor of five. However, our signal cross section is quartic in Yukawa couplings. If QCD corrections are of similar size as for single Higgs production, they could be absorbed by choosing a running $b$ mass at a scale intermediate between $m_{b}$ and $m_{H}$. Moreover, QCD corrections to the all-important squark loop contribu-

tions are not even known for the case of single Higgs production, if $m_{\tilde{q}} \lesssim \sqrt{\hat{s}} / 2$. Finally, our background calculation does not include (possibly quite large) QCD corrections, either. For these reasons, we prefer to conservatively quote results based on running Yukawa couplings taken at the large scale $Q$ introduced in the previous paragraph.

\section{SIGNAL AND BACKGROUND STUDY}

\section{A. Signal and Background Rates}

As well known, the masses of the CP-even Higgs bosons in the MSSM receive large radiative corrections, in particular from the top-stop sector [15]. We treat these corrections in the one-loop approximation, as calculated in the effective potential method [16]. We include leading two-loop corrections by choosing the appropriate scales for the running top 
quark mass when calculating the masses of the neutral Higgs bosons [19]. As in ref. [10], we take equal soft breaking contributions to diagonal entries of the stop and sbottom mass matrices $\left(m_{\tilde{t}_{L}}=m_{\tilde{t}_{R}}=m_{\tilde{b}_{R}} \equiv m_{\tilde{q}}\right)$, as well as equal trilinear soft breaking parameters in the stop and sbottom sectors $\left(A_{t}=A_{b} \equiv A_{q}\right)$. We fix the running masses of the top and bottom quarks to $m_{t}\left(m_{t}\right)=165 \mathrm{GeV}$ and $m_{b}\left(m_{b}\right)=4.2 \mathrm{GeV}$, respectively. This leaves us with a total of 5 free parameters which determine our signal cross sections: $m_{A}, \tan \beta, m_{\tilde{q}}, A_{q}$ and the supersymmetric higgsino mass parameter $\mu$.

Of course, this parameter space is subject to experimental constraints. We interpret the unsuccessful searches for Higgs bosons at LEP as implying the following constraints [20]:

$$
\begin{aligned}
m_{h} & >95 \mathrm{GeV}, \quad \text { if }|\sin (\alpha-\beta)|>0.6 ; \\
m_{h}+m_{A} & >180 \mathrm{GeV}, \quad \text { if }|\cos (\alpha-\beta)|>0.6 ; \\
m_{h} & >80 \mathrm{GeV} .
\end{aligned}
$$

Here, $\alpha$ is the mixing angle in the $\mathrm{CP}$-even Higgs sector; $\sin (\alpha-\beta)$ and $\cos (\alpha-\beta)$ determine the size of the $Z Z h$ and $Z A h$ couplings, respectively [21]. The third condition in (1) has been included to catch "pathological" cases. These constraints are only a rough approximation of the current search limits; however, a more elaborate treatment of the experimental constraints would not change our results significantly.

We also demand that the masses of the lighter physical stop and sbottom states satisfy

$$
m_{\tilde{t}_{1}}, m_{\tilde{b}_{1}}>90 \mathrm{GeV}
$$

which follows from squark searches at LEP [22]. We ignore constraints from stop and sbottom searches at the Tevatron, since these are stronger than (2) only for very large mass splitting between the squark mass eigenstates and the lightest superparticle (LSP). We also require that the contribution from stop and sbottom loops to the electroweak $\rho$-parameter [23] satisfies [24]

$$
\delta \rho_{\tilde{t} \tilde{b}} \leq 0.0017
$$

Finally, we only consider values of $A_{q}$ and $\mu$ in the range

$$
\left|A_{q}\right|,|\mu| \leq 3 m_{\tilde{q}} ;
$$

this is necessary to avoid the breaking of electric charge and color in the absolute minimum of the scalar potential [25].

As already mentioned, there are 6 different channels for producing two neutral Higgs bosons in the MSSM: $H H, h h, A A, H h, H A$ and $h A$. Often several channels contribute to a given signal even after cuts have been applied, once the experimental resolution has been taken into account. The reason is that often two Higgs bosons are essentially degenerate in mass, especially for high $\tan \beta$. For example, for heavy squarks and $\tan \beta \gg 1$, one has $m_{h} \simeq m_{A}$ for $m_{A} \lesssim 120 \mathrm{GeV}$ and $m_{H} \simeq m_{A}$ for $m_{A} \gtrsim 120 \mathrm{GeV}$. In the 'intermediate' region $\left(120 \mathrm{GeV} \lesssim m_{A} \lesssim 130 \mathrm{GeV}\right)$ all three neutral Higgs bosons are almost degenerate. This is illustrated in Fig. 1, which shows $\left(m_{h}, m_{H}\right)$ versus $m_{A}$ for three values of $\tan \beta$. In Fig. 1 we fixed $m_{\tilde{q}}, A_{q}$ and $\mu$ to $1 \mathrm{TeV}, 2.4 \mathrm{TeV}$ and 0 , respectively, corresponding to 'maximal' 
(stop) mixing. On the other hand, even at large $\tan \beta$ there are cases where this degeneracy is not very close, if $m_{A}$ is not large. This is illustrated by the heavy line in Fig. 1, where we have taken a set of parameters leading to large $\tilde{b}$ loop contributions to $h h$ production: $m_{\tilde{q}}=330 \mathrm{GeV}, A_{q}=700 \mathrm{GeV}, \mu=600 \mathrm{GeV}$ and $\tan \beta=50$. For this choice of parameters, $m_{h}$ is significantly smaller than $m_{A}$ everywhere, and $m_{H} \simeq m_{A}$ only for $m_{A} \gtrsim 250 \mathrm{GeV}$. 


\section{FIGURES}

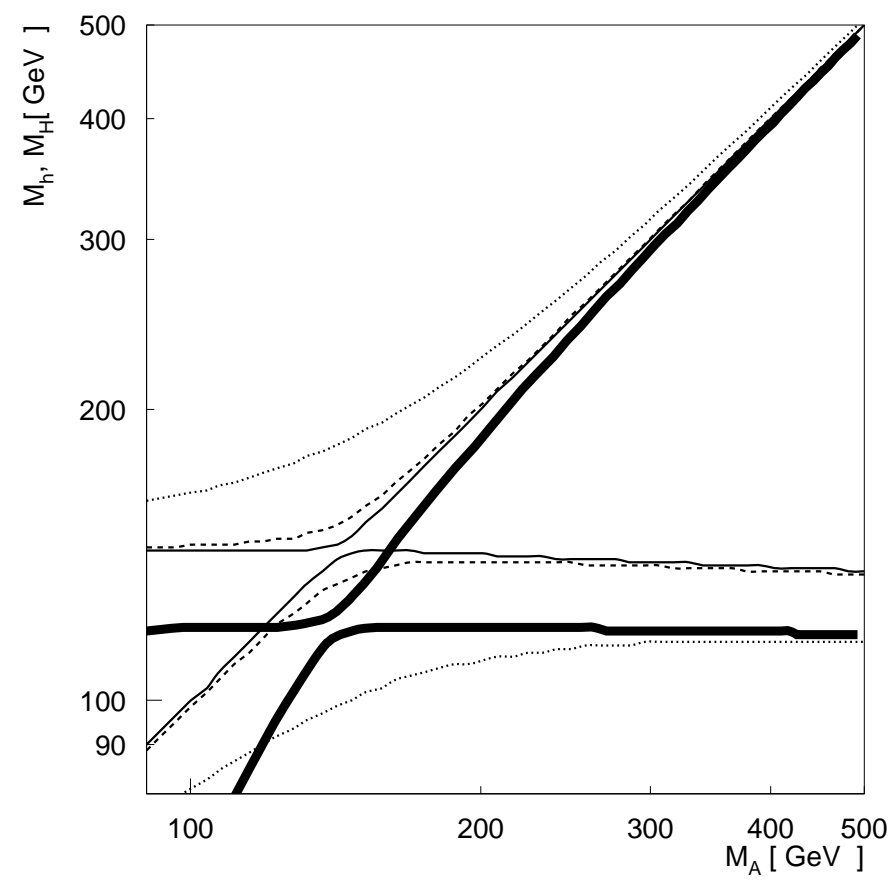

FIG. 1. $m_{h}$ and $m_{H}$ versus $m_{A}$ for $m_{\tilde{q}}=1 \mathrm{TeV}, A_{q}=2.4 \mathrm{TeV}, \mu=0$ and $\tan \beta=2$ (dotted), 9 (dashed) and 45 (thin solid), respectively. The heavy solid line is for $m_{\tilde{q}}=330 \mathrm{GeV}, A_{q}=700$ $\mathrm{GeV}, \mu=600 \mathrm{GeV}$ and $\tan \beta=50$. Note that we did not impose the Higgs search limits (1) here.

In our analysis we have combined contributions from different production channels assuming a Gaussian distribution for the reconstructed Higgs boson mass. We start with the diagonal process ( $h h, H H$ or $A A$ production) giving the best signal significance, and then add all other contributions to the "search window" defined below, after resolution smearing has been taken into account. In order to give an idea of the signal rate for negligible squark loop contributions, in Fig. 2 we present contours of constant total signal cross section in $\mathrm{fb}$ in the $\left(m_{A}, \tan \beta\right)$ plane. 

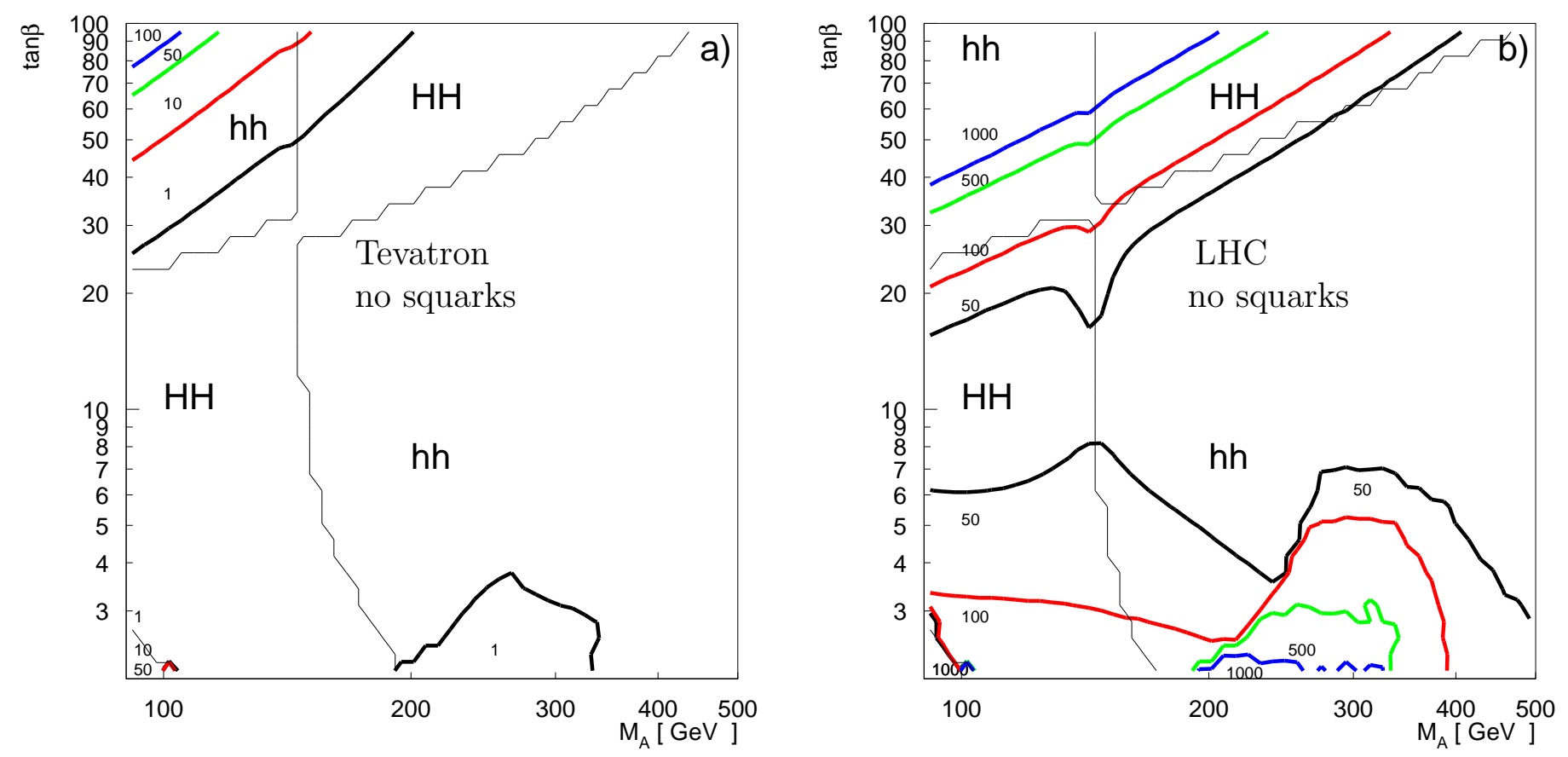

FIG. 2. Contours of constant cross section (in fb) for combined Higgs pair production channels, for the case of negligible squark loop contributions. Results for the Tevatron and the LHC are shown in frames (a) and (b), respectively.

The squark mass parameters used in Fig. 2 are the same as used for the thin lines in Fig. 1. Two of the three possible enhancement factors mentioned in the Introduction are clearly visible in this figure: the enhancement of $b$-quark loop contributions at large $\tan \beta$, where the cross section grows $\alpha \tan ^{4} \beta$; and the enhancement around $m_{A}=300 \mathrm{GeV}$ at small $\tan \beta$, from $H \rightarrow h h$ decays. The total cross section is about 200 times higher at the LHC than at the Tevatron. Given an integrated luminosity of $100 \mathrm{fb}^{-1}$, we expect well over 1,000 Higgs pair events at the LHC for all combinations of $m_{A}$ and $\tan \beta$. In contrast, if squark loop contributions are indeed small, at the Tevatron the raw signal rate is often too small to give a positive signal at Run II $\left(2 \mathrm{fb}^{-1}\right)$, or even at TeV33 $\left(25 \mathrm{fb}^{-1}\right)$, even if backgrounds were negligible.

The thin lines in Fig. 2 delineate regions where different channels dominate the total signal. For large $m_{A}$ and not very large $\tan \beta$, $h h$ production is always the most important channel. However, for $m_{A} \gtrsim 130 \mathrm{GeV}$ the enhancement of the $b$-quark Yukawa coupling is only felt by the heavy Higgs bosons $H$ and $A$, which are nearly degenerate at large $\tan \beta$; the production of these heavier states therefore dominates in this region. For smaller values of $m_{A}$, hh production is dominant at large $\tan \beta$, where the $h b \bar{b}$ coupling is proportional to $\tan \beta$. However, for $\tan \beta \lesssim 20$ and small $m_{A}, H H$ production dominates again, since the $h t \bar{t}$ coupling is suppressed $(\alpha \cot \beta)$.

In order to decide whether a Higgs pair cross section leads to a detectable signal, we have to compute the background rate. In order to suppress "fake" backgrounds, we require that all four $b$-jets are tagged as such. The total cross sections for the two main irreducible backgrounds, from $Z b \bar{b}$ production as well as pure QCD $b \bar{b} b \bar{b}$ production, for the basic 
parton-level acceptance cuts $p_{T}>25 \mathrm{GeV}, \Delta R_{j j}>0.5$ are:

$$
\begin{aligned}
\sigma[Z b \bar{b}]\left(Q=M_{Z}\right) & =1.5(59) \text { pb at the Tevatron (LHC); } \\
\sigma[b \bar{b} b \bar{b}]\left(Q=M_{b \bar{b}}\right) & =2.6(330) \text { pb at the Tevatron (LHC). }
\end{aligned}
$$

Note that these cross sections do not include any $b$-tagging efficiency. For the same acceptance cuts, and again ignoring $b$-tagging efficiencies, the cross sections for the most important "fake" backgrounds from $W b \bar{b}$ and especially $j j b \bar{b}$ production are:

$$
\begin{gathered}
\sigma[j j b \bar{b}]\left(Q=M_{b \bar{b}}\right)=1.6(164) \text { nb at the Tevatron (LHC); } \\
\sigma[W b \bar{b}]\left(Q=M_{W}\right)=3.1(19.1) \text { pb at the Tevatron (LHC). }
\end{gathered}
$$

Since the mis-tag probability of light quark and gluon jets is expected to be $\lesssim 1 \%$ [17, 27, after $b$-tagging these "fake" backgrounds are much smaller than the irreducible backgrounds listed above. We therefore ignore these "fake" backgrounds from now on.

The background cross sections quoted above only include contributions where the entire final state is produced in a single partonic collision. In particular at the LHC, there are also contributions where two independent partonic collisions can produce one $b \bar{b}$ pair each. The partons in the initial state can come either from the same $p p$ collision, or from independent collisions; recall that at full luminosity, one expects about 20 inelastic $p p$ interactions per bunch collision at the LHC. In the former case, the cross section can be estimated as $\sigma(g g, q \bar{q} \rightarrow b \bar{b})^{2} /\left(2 \pi R^{2}\right)$; here, $\pi R^{2} \simeq 15 \mathrm{mb}$ is the "effective area" of the proton [26]. We find that this contribution amounts to no more than $10 \%$ of the $2 \rightarrow 4$ pure QCD background, and can thus be ignored. In contrast, we found the $4 b$ background from two independent $p p$ interactions to be comparable to that from QCD $2 \rightarrow 4$ processes. However, good $b$-tagging requires a precise determination of both the primary vertex (from the partonic collision) and the secondary vertices (from $b$-decay). Background events where the two $b \bar{b}$ pairs come from independent $p p$ collisions should have two distinct "primary" vertices. This feature should allow to suppress these backgrounds efficiently. In the following we therefore only include irreducible backgrounds from a single partonic collision.

If only the basic acceptance cuts are used, these irreducible backgrounds are clearly far larger than the signal that can be achieved without sizable contribution from squark loops, see Fig. 2. A more elaborate set of cuts is thus necessary; this is the topic of the following subsection.

\section{B. Kinematical Analysis}

The signal consists of two pairs of $b$-jets. As already noted, we require all four $b$-jets to be tagged as such, in order to suppress "fake" backgrounds. A realistic description of the $b$-tagging efficiency is therefore very important. In case of the Tevatron, we use the projected $b$-tagging efficiency of the upgraded D $\varnothing$ detector [17]:

$$
\epsilon_{b}=0.57 \cdot \tanh \left(\frac{p_{T}}{35 \mathrm{GeV}}\right)
$$

where $p_{T}$ refers to the transverse momentum of the $b$-jet. For the LHC, we parameterize numerical results by the CMS collaboration [27]: 


$$
\epsilon_{b}= \begin{cases}0.6, & \text { for } p_{T}>100 \mathrm{GeV} \\ 0.1+p_{T} /(200 \mathrm{GeV}), & \text { for } 40 \mathrm{GeV} \leq p_{T} \leq 100 \mathrm{GeV} \\ 1.5 p_{T} /(100 \mathrm{GeV})-0.3, & \text { for } 25 \mathrm{GeV} \leq p_{T} \leq 40 \mathrm{GeV}\end{cases}
$$

We assume that $b$-jets can be tagged only for pseudorapidity $\left|\eta_{b}\right| \leq 2$ by both Tevatron and LHC experiments.

The jets in the signal are usually quite energetic, with average $p_{T}$ close to half the mass of the produced Higgs bosons. Since most of these bosons are produced fairly close to the kinematical threshold, the two jets in each pair are nearly back-to-back in the transverse plane. Moreover, since we focus on the production of two Higgs bosons with (nearly) identical mass, the two $b \bar{b}$ invariant masses should be equal within errors. These expectations are borne out by our Monte Carlo simulation. In Fig. Ba-f we show several kinematical distributions of the signal, compared with the $Z b \bar{b}$ (left column) and pure QCD $4 b$ background (right column). The signal has been computed for a Higgs boson mass of $120 \mathrm{GeV}$, assuming negligible contributions from squark loops and a large value of $\tan \beta$ (so that the signal is mostly due to $b$-quark loops). If the main contribution to the signal cross section comes from loops of heavier (s)particles the difference between signal and background becomes even more pronounced, since the signal is shifted towards somewhat larger values of $\hat{s}$, and hence has harder $p_{T}$ spectra. The results shown in these figures are not normalized, but include the $b$-tagging efficiency as parameterized in eq.(5). In detail, we constructed the following kinematical variables and respective set of cuts for an efficient extraction of the signal:

- Reconstructed Higgs boson mass, $M_{H}$ : since we do not attempt to distinguish $b$-jets from $\bar{b}$-jets, there are three possible ways to pair up the four $b$ (anti-)quarks in the final state. We chose the pairing that gives the smallest difference between the invariant masses of the two pairs. The reconstructed Higgs boson mass is then defined as $M_{H}=\left[M_{b_{1} b_{2}}+M_{b_{3} b_{4}}\right] / 2$. After resolution smearing, the distribution in $M_{H}$ for the signal can be described by a Gaussian with width $\sigma \simeq \sqrt{M_{H}}$ (in GeV units), supplemented by a tail towards small values due to hard out-of-cone radiation, as well as energy lost in neutrinos produced in the decay of the $b$-quarks. The same effects also shift the peak of the Gaussian downwards by about $10 \%$, as compared to the physical (input) Higgs boson mass $m_{H, i n}$. This is illustrated in Fig. 3a. We thus define our search window in the reconstructed Higgs boson mass as

$$
0.9 m_{H, i n}-1.5 \sigma \leq M_{H} \leq 0.9 m_{H, i n}+1.5 \sigma \text {. }
$$

- The mass difference between the invariant masses of the two pairs should be small, see Fig. 3b. We thus demand

$$
\Delta M_{H}=\left|M_{b_{1} b_{2}}-M_{b_{3} b_{4}}\right| \leq 2 \sigma \text {. }
$$

- The angles in the transverse plane between the two jets in each pair should be large:

$$
\Delta \phi_{b_{1}, b_{2}}, \Delta \phi_{b_{3}, b_{4}}>1
$$


Another feature of the signal is that the two Higgs bosons have similar $p_{T}$ (it would be equal in the absence of initial state radiation), and hence also similar transverse velocities (or boosts). The two transverse opening angles therefore tend to be correlated, so that the difference between them is small. We can thus require:

$$
\left|\Delta \phi_{b_{1}, b_{2}}-\Delta \phi_{b_{3}, b_{4}}\right|<1
$$

These cuts are illustrated in Fig. 3ic.

- All four $b$-jets in the signal are fairly hard. We nevertheless found it advantageous to use slightly different cuts for the softest and hardest of these jets, with transverse momenta $p_{T, \min }$ and $p_{T, \max }$, respectively, see Figs. $3 \mathrm{~d} \mathrm{~d}, \mathrm{e}$ :

$$
\begin{gathered}
\text { Tevatron }: p_{T, \min }>M_{H} / 8+1.25 \sigma ; p_{T, \max }>M_{H} / 8+2 \sigma . \\
\mathrm{LHC}: p_{T, \min }>M_{H} / 4 ; p_{T, \max }>M_{H} / 4+2 \sigma .
\end{gathered}
$$

We use harder cuts for the LHC since here it is important to increase not only the significance but also the signal to background ratio. For the large number of events expected at the LHC, systematic errors will play an important role in the observability of the signal; conclusions based on an analysis of the statistical significance only may therefore be misleading.

- The $4 b$ invariant mass $M_{4 b}$ was also found to be important for two reasons. First, the signal distribution for this variable is concentrated around the invariant mass of the Higgs pair, which is significantly larger than the kinematic minimum implied by the cuts (11) on the transverse momenta of the four $b$-jets; see Fig. 3f. We therefore require:

$$
M_{4 b}>1.9 M_{H}-3 \sigma
$$

Moreover, this quantity has been shown to be useful for disentangling quark and squark loop contributions [10]. Different masses of the particles in the loop lead to different form factors, and hence to different dependence on the partonic center-of-mass energy $\sqrt{\hat{s}} \simeq M_{4 b}$. 

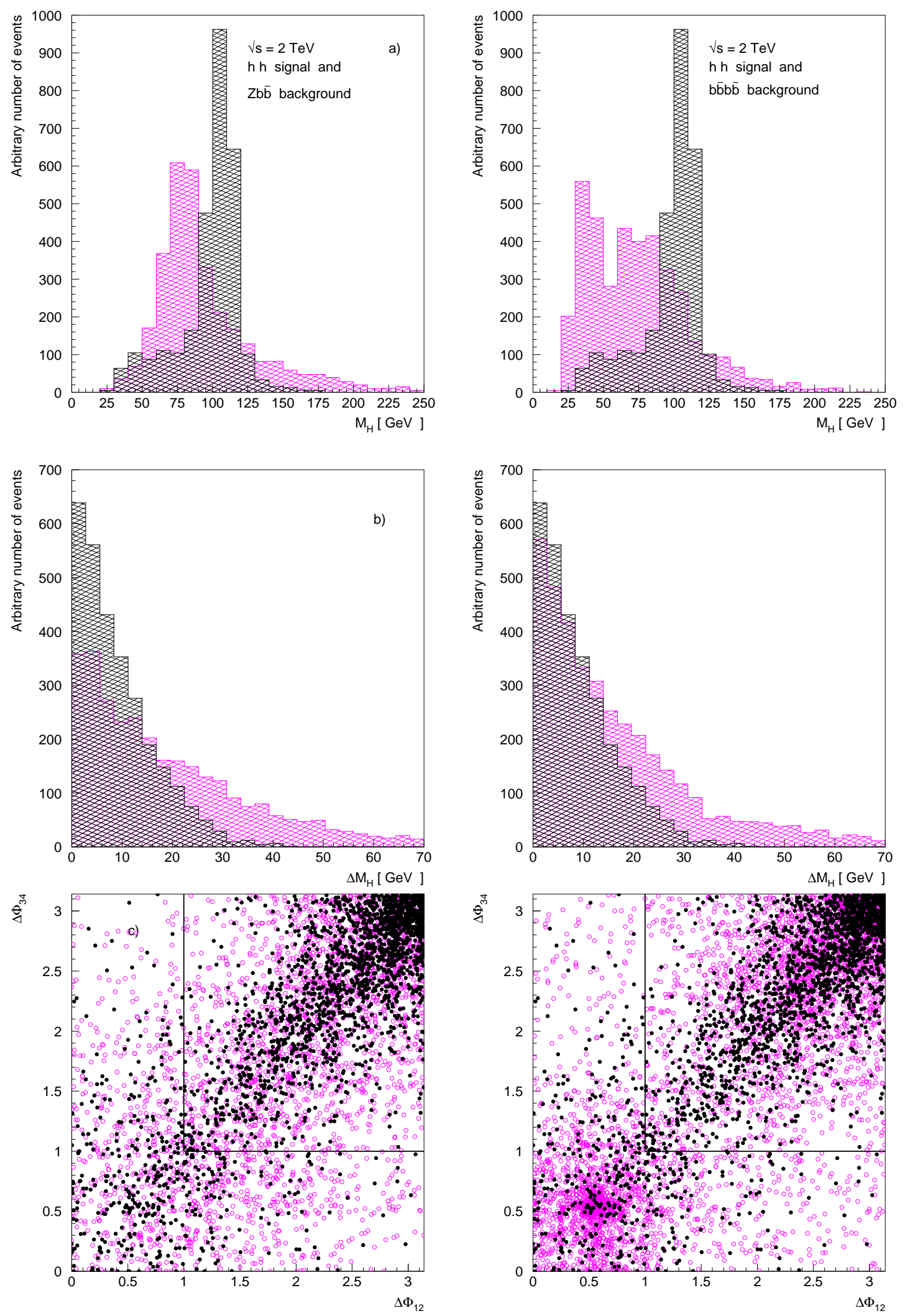

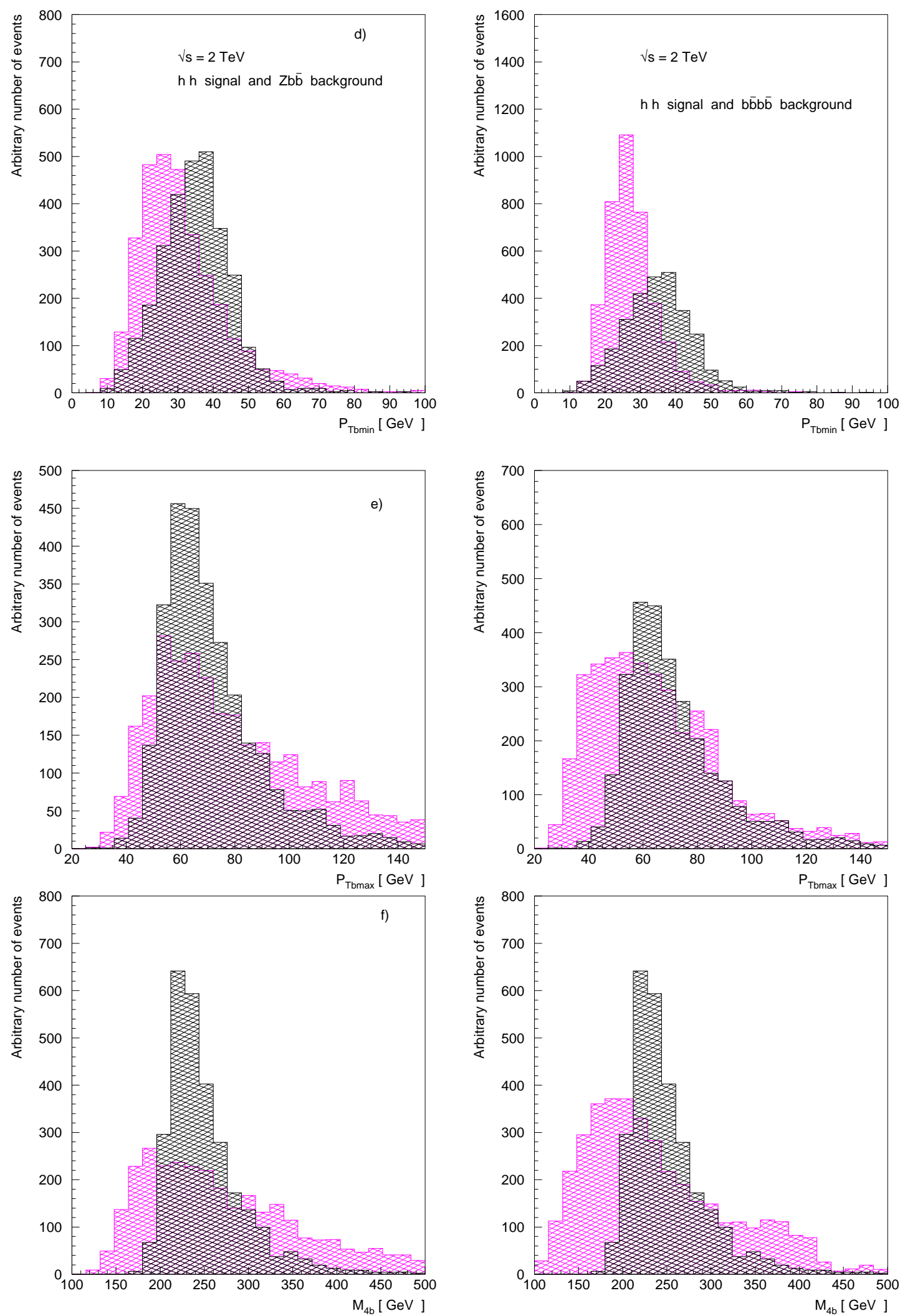

FIG. 3. Various kinematic distributions of the signal for the production of a pair of Higgs bosons with mass $120 \mathrm{GeV}$ (dark histograms and circles), compared to the $Z b \bar{b}$ background (left column) and the pure QCD $4 b$ background (right column). These results are for the Tevatron; the distributions for the LHC look similar. 
The efficiency of these cuts for various input (search) Higgs boson masses is listed in the following Tables for the Tevatron and LHC, respectively. We have used the following sequence of cuts:

cut 1: $p_{T, \min }, p_{T, \max }$, and pseudorapidity $\left|\eta_{b}\right| \leq 2$.

cut 2 : cut $1+\Delta M_{H}$.

cut 3: cut $2+\Delta \phi$.

cut 4 : cut $3+$ mass window $+M_{4 b}$.

In these Tables we have applied $b$-tagging after all the other cuts. The signal efficiency refers to the total Higgs pair cross section times branching ratio for the $4 b$ final state. The background efficiency refers to the background cross section defined through the basic acceptance cuts $\left(p_{T}(b)>25 \mathrm{GeV}\right.$ for all four $b$ (anti-)quarks, and jet separation $\Delta R_{j j}>0.5$ for all jet pairs). 


\section{TABLES}

\begin{tabular}{|c|c|c|c|c|c|c|c|}
\hline$m_{H, i n}[\mathrm{GeV}]$ & & 100 & 120 & 140 & 160 & 180 & 200 \\
\hline$\epsilon_{\text {signal }}[\%]$ & \multirow{3}{*}{ cut 1} & 51 & 56 & 59 & 62 & 65 & 67 \\
\hline$\epsilon_{Z b b}[\%]$ & & 40.1 & 27.5 & 18.1 & 12.5 & 8.9 & 6.0 \\
\hline$\epsilon_{b b b b}[\%]$ & & 38.6 & 19.4 & 8.8 & 4.6 & 2.3 & 1.4 \\
\hline$\epsilon_{\text {signal }}[\%]$ & \multirow{3}{*}{ cut 2} & 49 & 53 & 55 & 56 & 58 & 57 \\
\hline$\epsilon_{Z b b}[\%]$ & & 25.6 & 17.6 & 11.7 & 7.8 & 5.6 & 3.8 \\
\hline$\epsilon_{b b b b}[\%]$ & & 26.3 & 13.1 & 5.86 & 3.08 & 1.52 & .92 \\
\hline$\epsilon_{\text {signal }}[\%]$ & \multirow{3}{*}{ cut 3} & 40 & 44 & 45 & 46 & 48 & 47 \\
\hline$\epsilon_{Z b b}[\%]$ & & 18.3 & 12.5 & 8.0 & 5.0 & 3.4 & 2.3 \\
\hline$\epsilon_{b b b b}[\%]$ & & 14.1 & 7.04 & 3.36 & 1.77 & .87 & .55 \\
\hline$\epsilon_{\text {signal }}[\%]$ & \multirow{3}{*}{ cut 4} & 32 & 35 & 36 & 34 & 36 & 36 \\
\hline$\epsilon_{Z b b}[\%]$ & & 9.87 & 3.17 & 1.94 & 1.22 & .67 & .35 \\
\hline$\epsilon_{b b b b}[\%]$ & & 7.41 & 2.71 & .761 & .460 & .195 & .088 \\
\hline$\epsilon_{\text {signal }}[\%]$ & \multirow{3}{*}{$4 b$ tag } & 1.44 & 2.10 & 2.53 & 2.74 & 3.17 & 3.30 \\
\hline$\epsilon_{Z b b}[\%]$ & & .460 & .187 & .133 & .0935 & .0541 & .0314 \\
\hline$\epsilon_{b b b b}[\%]$ & & .292 & .137 & .049 & .0318 & .0153 & .0072 \\
\hline$b b b b+Z b b$ & \# events & 17.5 & 8.1 & 3.2 & 2.1 & 1.1 & .5 \\
\hline$Z b b$ & for & 2.2 & .9 & .6 & .4 & .3 & .1 \\
\hline$b b b b$ & $2 \mathrm{fb}^{-1}$ & 15.3 & 7.2 & 2.6 & 1.7 & .8 & .4 \\
\hline signal $[\mathrm{fb}] \times \operatorname{Br}(4 b)$ & $95 \%$ c.l. & 280 & 153 & 93.8 & 78.4 & 59.0 & 45.3 \\
\hline signal $[\mathrm{fb}] \times \operatorname{Br}(4 b)$ & $5 \sigma$ & 575 & 413 & 268 & 229 & 179 & 148 \\
\hline$b b b b+Z b b$ & \# events & 219 & 101 & 40.2 & 26.3 & 13.2 & 6.6 \\
\hline$Z b b$ & for & 27.2 & 11.1 & 7.9 & 5.5 & 3.2 & 1.9 \\
\hline$b b b b$ & $25 \mathrm{fb}^{-1}$ & 192 & 89.9 & 32.3 & 20.8 & 10.0 & 4.7 \\
\hline signal $[\mathrm{fb}] \times B r(4 b)$ & $95 \%$ c.l. & 80.2 & 37.4 & 19.6 & 14.6 & 9.7 & 7.1 \\
\hline signal $[\mathrm{fb}] \times B r(4 b)$ & $5 \sigma$ & 165 & 76.9 & 40.2 & 30.0 & 25.5 & 19.4 \\
\hline
\end{tabular}

TABLE I. Signal and background efficiencies and minimal cross sections for a $95 \%$ c.l. exclusion limit on, as well as a $5 \sigma$ discovery of, Higgs boson pair production at the Tevatron, for several values of the "input" Higgs boson mass $m_{H, i n}$. See the text for a detailed description of the cuts. 


\begin{tabular}{|c|c|c|c|c|c|c|c|}
\hline$m_{H, i n}[\mathrm{GeV}]$ & & 100 & 12120 & 14140 & 160 & 180 & 200 \\
\hline$\epsilon_{\text {signal }}[\%]$ & \multirow{3}{*}{ cut 1} & 32 & 33 & 35 & 38 & 35 & 38 \\
\hline$\epsilon_{Z b b}[\%]$ & & 25.1 & 14.5 & 9.0 & 5.7 & 3.9 & 2.8 \\
\hline$\epsilon_{b b b b}[\%]$ & & 19.4 & 10.2 & 5.4 & 3.1 & 2.0 & 1.1 \\
\hline$\epsilon_{\text {signal }}[\%]$ & \multirow{3}{*}{ cut 2} & 30 & 30 & 32 & 34 & 30 & 33 \\
\hline$\epsilon_{Z b b}[\%]$ & & 11.7 & 6.2 & 3.5 & 2.1 & 1.3 & 1.0 \\
\hline$\epsilon_{b b b b}[\%]$ & & 10.2 & 5.08 & 2.85 & 1.51 & .93 & .56 \\
\hline$\epsilon_{\text {signal }}[\%]$ & \multirow{3}{*}{ cut 3} & 23 & 23 & 26 & 27 & 23 & 26 \\
\hline$\epsilon_{Z b b}[\%]$ & & 7.8 & 4.1 & 2.2 & 1.2 & .7 & .4 \\
\hline$\epsilon_{b b b b}[\%]$ & & 5.47 & 2.83 & 1.46 & .83 & .54 & .30 \\
\hline$\epsilon_{\text {signal }}[\%]$ & \multirow{3}{*}{ cut 4} & 19 & 19 & 20 & 21 & 18. & 19 \\
\hline$\epsilon_{Z b b}[\%]$ & & 4.23 & 1.71 & .70 & .56 & .14 & .10 \\
\hline$\epsilon_{b b b b}[\%]$ & & 2.30 & .965 & .508 & .271 & .288 & .119 \\
\hline$\epsilon_{\text {signal }}[\%]$ & \multirow{3}{*}{$4 b$ tag } & .16 & .34 & .60 & .90 & 1.02 & 1.38 \\
\hline$\epsilon_{Z b b}[\%]$ & & .0334 & .0263 & .0187 & .0190 & .0054 & .0081 \\
\hline$\epsilon_{b b b b}[\%]$ & & .0139 & .0142 & .0127 & .0112 & .0107 & .0071 \\
\hline $\mid b b b b+Z b b$ & \# \# events & 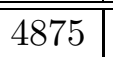 & 49900 & 4337 & 3863 & 3501 & 2419 \\
\hline$Z b b$ & for & 305 & 240 & 171 & 174 & 49.0 & 73.6 \\
\hline$b b b b$ & $100 \mathrm{fb}^{-1}$ & 4570 & 4660 & 4166 & 3689 & 3452 & 2345 \\
\hline signal $[\mathrm{fb}] \times B r(4 b)$ & 95\% c.l. & 1212 & 570 & 290 & 171 & 118 & 70.1 \\
\hline signal $[\mathrm{fb}] \times \operatorname{Br}(4 b)$ & $5 \sigma$ & 3030 & 1426 & 726 & 427 & 296 & 175 \\
\hline
\end{tabular}

TABLE II. Signal and background efficiencies and minimal cross sections for a $95 \%$ c.l. exclusion limit on, as well as a $5 \sigma$ discovery of, Higgs boson pair production at the LHC, for several values of the "input" Higgs boson mass $m_{H, i n}$. See the text for a detailed description of the cuts. 
We note that before the $b$-tagging efficiency is included, the signal efficiency is essentially independent of the Higgs boson mass. At this stage the signal efficiency is nearly two times higher at the Tevatron than at the LHC, due to the stronger $p_{T}$ cuts applied at the latter. Again before $b$-tagging, the efficiency of the $Z b \bar{b}$ and especially of the pure QCD $4 b$ background decreases very quickly with increasing "input" Higgs boson mass, due to the rather soft $p_{T}$ spectra of these backgrounds. However, after the $p_{T}$-dependent efficiency for tagging all four $b$ (anti-)quarks has been factored in, the final signal efficiency increases significantly with increasing Higgs boson mass, whereas the efficiency for the dominant (pure QCD) background at the LHC becomes almost independent of $m_{H, \text { in }}$ as long as $m_{H, \text { in }} \lesssim 150$ $\mathrm{GeV}$. Note also that the final signal efficiency at the LHC is a factor 3 to 9 smaller than at the Tevatron, largely due to the worse $b$-tagging efficiency at moderate values of $p_{T}$ [cf. eqs.(5) and (6)].

These Tables also contain results for the minimal total signal cross section times branching ratio needed to exclude Higgs boson pair production at the $95 \%$ c.l., as well as the minimal total cross section times branching ratio required to claim a $5 \sigma$ discovery of Higgs boson pair production in the $4 b$ final state. We give these critical cross sections for two values of the integrated luminosity at the Tevatron, characteristic for the upcoming Run II and for the final luminosity at the end of the "TeV33" run, respectively. In case of the LHC, we give results for an integrated luminosity of $100 \mathrm{fb}^{-1}$, corresponding to one year of data at full luminosity.

As already mentioned, systematic uncertainties are a concern especially at the LHC, where the large signal rate can lead to a very small signal to background ratio if the significance is defined using statistical errors only. It can be argued that systematic uncertainties should be small, since the signal should manifest itself as a peak in the $M_{H}$ distribution on top of a smooth background, the size of which can be determined from side bins. However, given the rather large width of the peak, the number of available side bins will in practice be rather limited. We therefore assign an ad-hoc systematic uncertainty of $2 \%$ on the background estimate, as obtained by extrapolation from the side bins. We thus require a minimal signal to background ratio of 0.04 for the $95 \%$ c.l. exclusion limit, and 0.1 for the $5 \sigma$ discovery cross section. This requirement in fact fixes the critical cross sections at the LHC for $m_{H, \text { in }} \leq 180 \mathrm{GeV}$.

The signal efficiencies in these Tables have been computed under the assumption that the signal mostly comes from $b$-quark loops. Loops of heavier (s)particles lead to harder $\hat{s}$ distributions [10], and thus to harder $p_{T}$ spectra of the Higgs bosons. This has several effects. The average transverse momentum of the $b$-jets will increase, increasing the tagging efficiency as well as the efficiency of the $p_{T, \max }$ cut. On the other hand, the spectrum of the softest $b$-jet actually becomes softer, since the probability of substantial cancelations between the $b$-momentum in the Higgs rest frame and the boost into the lab frame increases with increasing momentum of the Higgs bosons. Moreover, the average opening angle between the $b$-jets within each pair becomes smaller, reducing the efficiency of the angular cuts (9) and (10). For the given set of cuts the net effect is to decrease the signal efficiency at the Tevatron by at most $25 \%$, while it remains more or less unchanged at the LHC. However, the correct procedure would be to re-optimize the cuts for the case that the signal is dominated by loops of heavy (s)particles (top quarks or $\tilde{t}_{1}$ or $\tilde{b}_{1}$ squarks). For example, one should choose even more asymmetric cuts on $p_{T, \min }$ and $p_{T, \text { max }}$, such that the background 
efficiency remains essentially the same. The final signal efficiency should then be at least as high as that shown in the Tables. Given the uncertainties of our calculation, we simply assume that the efficiencies, and hence the minimal cross sections for $95 \%$ exclusion and $5 \sigma$ discovery, are as listed in the Tables, independent of the mass of the (s)particle giving the dominant contribution to the signal.

Finally, it should be noted that we did not include trigger efficiencies in our calculation. This does not pose a problem for Tevatron experiments, where a simple 4-jet trigger should be sufficient, given our $p_{T}$-cuts (11a). However, LHC experiments will need far higher $p_{T}$ trigger thresholds for generic 4-jet events; e.g., ATLAS foresees a threshold of $90 \mathrm{GeV}$ per jet [28], which would remove most of our signal. On the other hand, (multi) $-b$ triggers are now being studied by the LHC experiments. We assume that these will indeed be implemented, and will allow to trigger on our signal events without significant loss of efficiency.

\section{POTENTIAL OF HADRON COLLIDERS FOR HIGGS PAIR SEARCH}

We are now in a position to estimate the Higgs boson pair production discovery reach of the Tevatron and the LHC. By comparing the results of Table 1 and Fig. 2a, it becomes clear that in the absence of sizable squark loop contributions to the signal cross section, the potential of Tevatron experiments for this search is essentially nil. In contrast, some parts of the $\left(m_{A}, \tan \beta\right)$ plane can be covered at the LHC even if squark loop contributions are negligible. This is illustrated in Fig. \&. Even with $25 \mathrm{fb}^{-1}$ of data, Tevatron experiments can only exclude a small sliver of parameter space with $\tan \beta>80$, well beyond the upper limit on this quantity in most implementations of the MSSM $\left(\tan \beta \lesssim m_{t}\left(m_{t}\right) / m_{b}\left(m_{t}\right) \simeq 60\right)$. In contrast, even for this pessimistic assumption of negligible squark loop contributions, LHC experiments might discover a $5 \sigma$ signal if $\tan \beta$ is large $(\gtrsim 50)$, and can at least exclude some regions of parameter space where $\tan \beta$ is small $(\lesssim 2.5)$. Note that our cuts are not optimized to search for $H \rightarrow h h \rightarrow b \bar{b} b \bar{b}$ events. By requiring that $M_{4 b}$ lies in a narrow window around $m_{H}$, in addition to cuts similar to those employed by us, the ATLAS collaboration finds [28] $\mathrm{a} \geq 5 \sigma$ signal (with $300 \mathrm{fb}^{-1}$ of data) for $\tan \beta \leq 3$, for the case $m_{H}=300 \mathrm{GeV}$.

\footnotetext{
${ }^{1}$ Their background calculation is based on the use of hard $2 \rightarrow 2$ matrix elements, mostly from $g g \rightarrow g g$, followed by parton showering. Our calculation, which is based on full $2 \rightarrow 4$ matrix elements, should be more accurate, and might lead to quantitative changes of the discovery region for $H \rightarrow h h$ events in the $4 b$ final state. It should nevertheless be clear that the presence of a peak in the $M_{4 b}$ distribution should make it easier to look for resonant $h h$ production than to search for the continuum events which are the main focus of our analysis.
} 

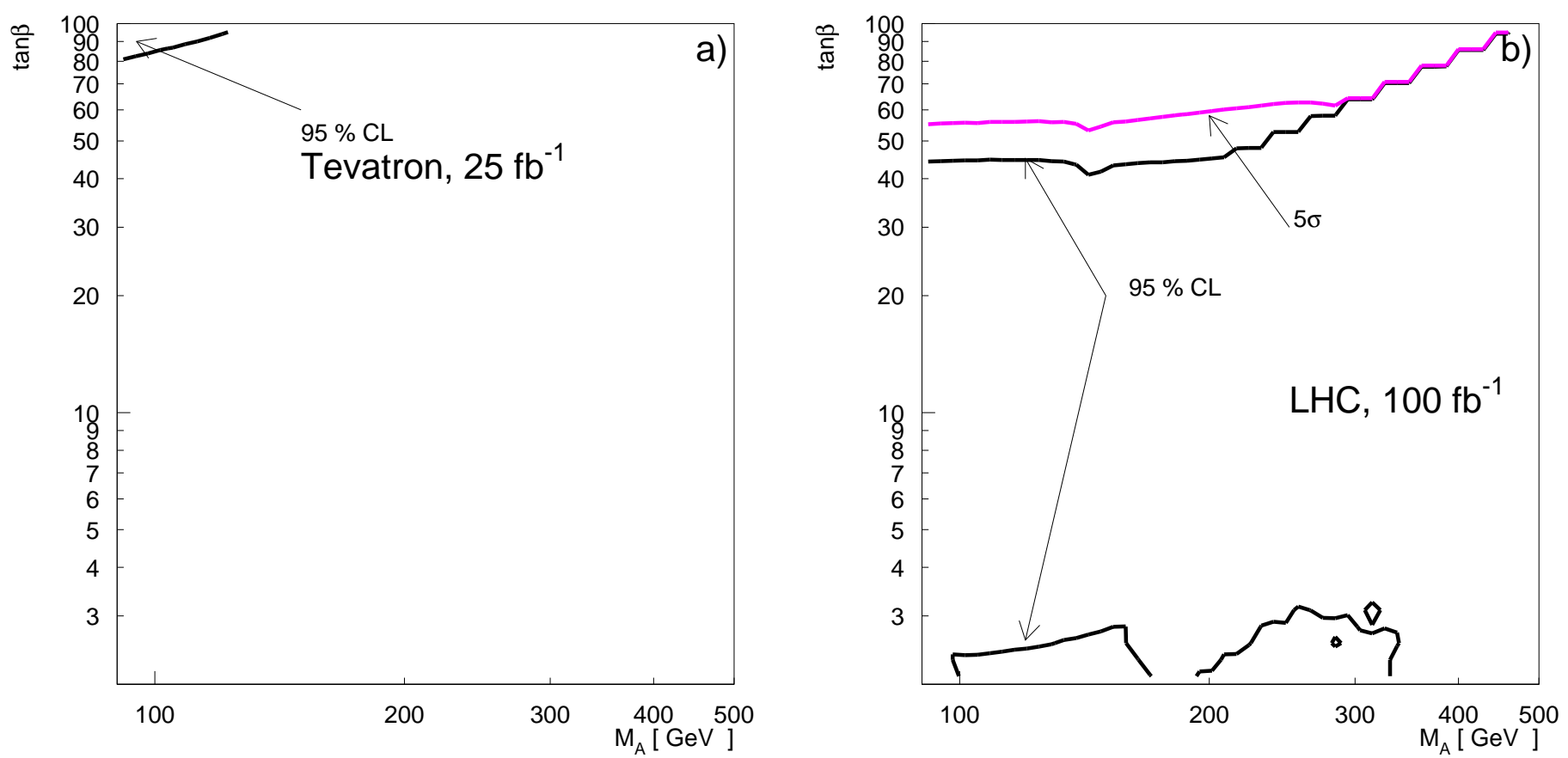

FIG. 4. 95\% c.l. exclusion and $5 \sigma$ discovery regions for Higgs boson pair production at the Tevatron with $25 \mathrm{fb}^{-1}$ of data (a), and the LHC with $100 \mathrm{fb}^{-1}$ of data (b), for the case of negligible squark loop contributions.

In ref. [10 it was found that the contribution from squark loops can exceed that from quark loops by more than two orders of magnitude. The prospects for Higgs pair searches therefore obviously depend crucially on the parameters of the squark sector, in addition to the parameters $m_{A}$ and $\tan \beta$ that describe the MSSM Higgs sector at the tree level. As already stated in the Introduction, squark loop contributions are large if the relevant lighter squark mass eigenstate $\left(\tilde{t}_{1}\right.$ or $\left.\tilde{b}_{1}\right)$ is light and the trilinear coupling of this mass eigenstate to Higgs bosons is large.

In order to illustrate the possible importance of squark loop contributions, we performed three different Monte Carlo searches of the three-dimensional parameter space $\left(m_{\tilde{q}}, A_{q}, \mu\right)$ describing squark masses and couplings under the assumptions discussed at the beginning of Sec. III. In the first and second search we maximize the square of the coupling of $h$ and $H$, respectively, to $\tilde{t}_{1}$ (at small $\tan \beta$ ) or $\tilde{b}_{1}$ (at large $\tan \beta$ ), divided by some power of the squark mass, and multiplied with the branching ratio of the Higgs boson in question into $b \bar{b}$; the quantity to be maximized is designed to closely track the complete numerical result for the squared squark loop contribution to $h h$ and $H H$ production, respectively. In the third search, which is relevant only for rather small values of $\tan \beta$, we maximize the squared $H \tilde{t}_{1} \tilde{t}_{1}$ coupling multiplied with $\operatorname{Br}(H \rightarrow h h) \cdot \operatorname{Br}^{2}(h \rightarrow b \bar{b})$ and divided by some power of $m_{\tilde{t}_{1}}$; here the quantity to be maximized approximates the squark loop contribution to single $H$ production, followed by on-shell $H \rightarrow h h$ and $h \rightarrow b \bar{b}$ decays.

These searches were performed for 500 combinations of $m_{A}$ and $\tan \beta$, placed on a grid with logarithmic spacing. For each point on this grid we computed the total cross sections corresponding to the three sets of input parameters found by the three Monte Carlo searches, at both the Tevatron and the LHC. Finally, we chose the set of input parameters giving the 
most significant signal for Higgs boson pair production. The result does not quite represent the most optimal situation, since the quantities maximized in the Monte Carlo search do not completely reproduce the behavior of various contributions to the total cross section; also, possible interference effects are difficult to include in such a procedure. Unfortunately, the numerical calculation of the 6 relevant Higgs pair production cross sections for a single set of input parameters takes more than two CPU minutes on an ALPHA-station; a Monte Carlo maximization of the full numerically computed cross section was therefore not possible with the available resources. However, we believe that our procedure should reproduce the maximal cross section compatible with the constraints (1) -(耳) to within a factor of two or so.

The results are presented in Fig. 5, which shows the regions that can be probed with 2 and $25 \mathrm{fb}^{-1}$ of data at the Tevatron (a), and with $100 \mathrm{fb}^{-1}$ of data at the LHC (b). We see that now virtually the entire part of the $\left(m_{A}, \tan \beta\right)$ plane still allowed by the LEP constraints (11) will give $\mathrm{a} \geq 5 \sigma$ signal at the LHC. Moreover, the entire region $m_{A} \leq 200$ $\mathrm{GeV}$, and most of the region with $m_{A} \leq 300 \mathrm{GeV}$, can be probed at the Tevatron with 25 $\mathrm{fb}^{-1}$ of data. Perhaps, the most surprising, and encouraging, result is that a substantial region of parameter space will give a $\geq 5 \sigma$ signal at the Tevatron already with $2 \mathrm{fb}^{-1}$ of data! This occurs e.g. for $m_{A}=160 \mathrm{GeV}, \tan \beta=10, m_{\tilde{q}}=410 \mathrm{GeV}, A_{q}=1.15 \mathrm{TeV}$ and $\mu=1 \mathrm{TeV}$, leading to a combined $(h h, H H, A A, h H, h A, H A) \rightarrow 4 b$ cross section of $3.3 \mathrm{pb}$. Another example occurs for $m_{A}=170 \mathrm{GeV}, \tan \beta=29, m_{\tilde{q}}=326 \mathrm{GeV}, A_{q}=0.78 \mathrm{TeV}$ and $\mu=0.98 \mathrm{TeV}$, which gives a signal cross section (before cuts) of $4.7 \mathrm{pb}$. This is the first time that such a robust signal for Higgs boson production at the next run of the Tevatron collider has been suggested.
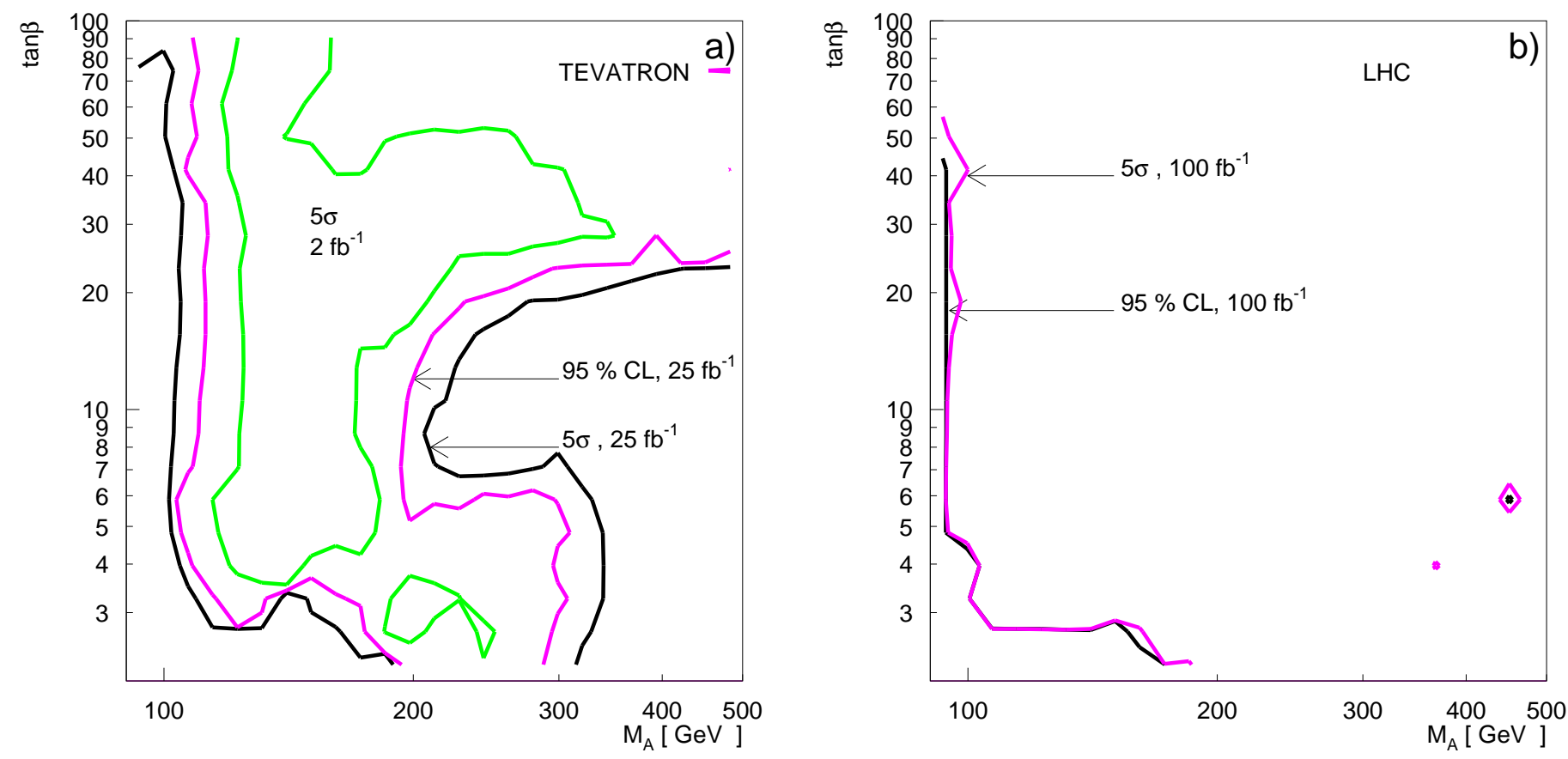
FIG. 5. 95\% c.l. exclusion and $5 \sigma$ discovery regions for Higgs pair production at the Tevatron $\left(25 \mathrm{fb}^{-1}\right)(\mathrm{a})$ and LHC $\left(100 \mathrm{fb}^{-1}\right)(\mathrm{b})$, for "maximized" squark loop contributions (see the text for details). The light grey contour in (a) shows the region where a $\geq 5 \sigma$ signal should be detectable at the Tevatron with just $2 \mathrm{fb}^{-1}$ of data.

Our search strategy attempts to maximize the signal for Higgs boson pair production for given values of $m_{A}$ and $\tan \beta$. This is not the same as maximizing this signal for fixed mass $m_{h}$ of the light $\mathrm{CP}$-even scalar Higgs boson. Nevertheless our search of the squark parameter space also yielded significantly larger maximal signal cross sections for fixed $m_{h}$, as compared to the case of negligible squark loop contribution depicted in Fig. 2. This is illustrated in Fig. 6, which shows the maximal signal cross section (before cuts) at the LHC found by our scans of parameter space, as a function of $m_{h}$. Attempts to maximize the squark loop contribution to the signal typically yield $m_{\tilde{q}} \lesssim 400 \mathrm{GeV}$; moreover, the choices of $A_{q}$ and $\mu$ are different from that that maximize $m_{h}$. Scenarios with large squark loop contribution therefore have significantly smaller $m_{h}$ for given $m_{A}$ and $\tan \beta$. This explains why the upper curve in Fig. 6 already terminates at $m_{h}=120 \mathrm{GeV}$, while the lower curve, which is for the same parameters as Fig. 2, extends to $130 \mathrm{GeV}$. This also explains why the enhancement due to squark loops becomes smaller as $m_{h}$ increases. We should emphasize that cases with significantly larger enhancement of the signal due to squark loops may well exist, since, as already noted, our search strategy was not optimized for maximizing this enhancement for a fixed value of $m_{h}$. Nevertheless the qualitative behavior of the true maximum of the signal should be similar to that depicted in Fig. 6.

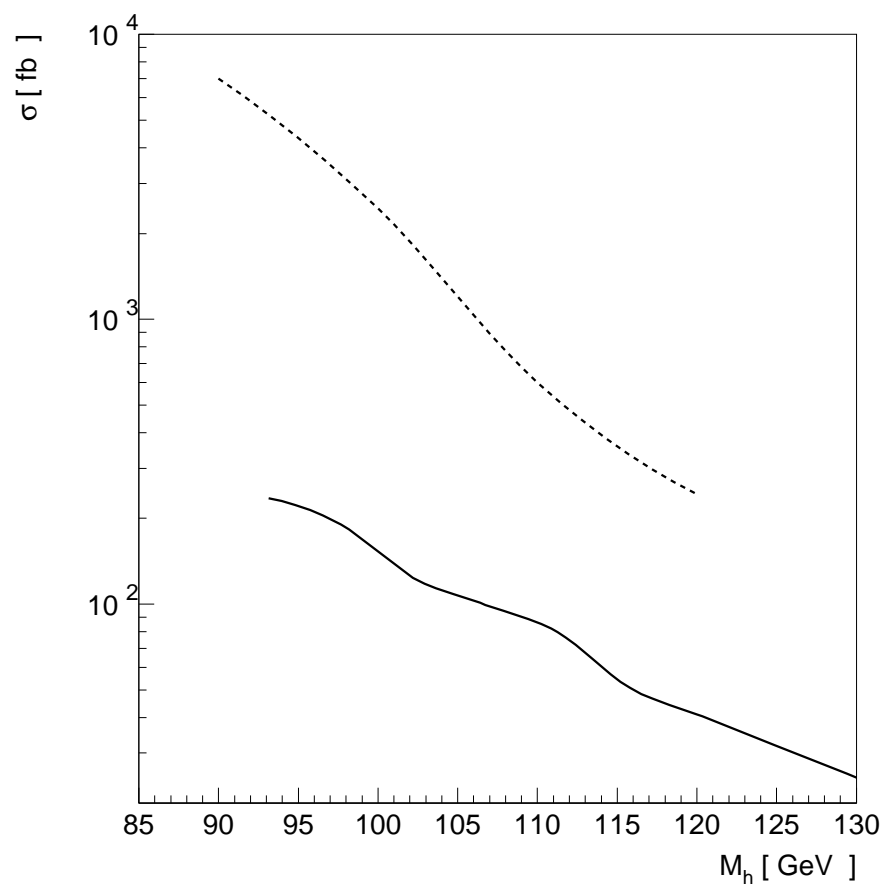

FIG. 6. Maximal value of the total cross section for a given value of $m_{h}$ for the case of heavy squarks (lower curve), and for the case of "maximal" enhancement due to squarks (upper curve).

As already noted, the results shown in Figs. 5 and 6 show the most significant signal channel. We found that, unlike for the case of negligible squark loop contribution, the 
most significant signal now always comes from $h$ h production, in some cases augmented by the production of nearly degenerate Higgs bosons ( $h A$ and $A A$ production); however, these auxiliary modes contribute much less to the total signal, since squark loop contributions to these modes are absent (for the $h A$ channel) or relatively small (for $A A$ production). It is then interesting to investigate the question whether the production of heavier Higgs bosons, in particular $\mathrm{HH}$ production (augmented again by nearly degenerate modes), might also yield a (less significant, but still) observable signal.
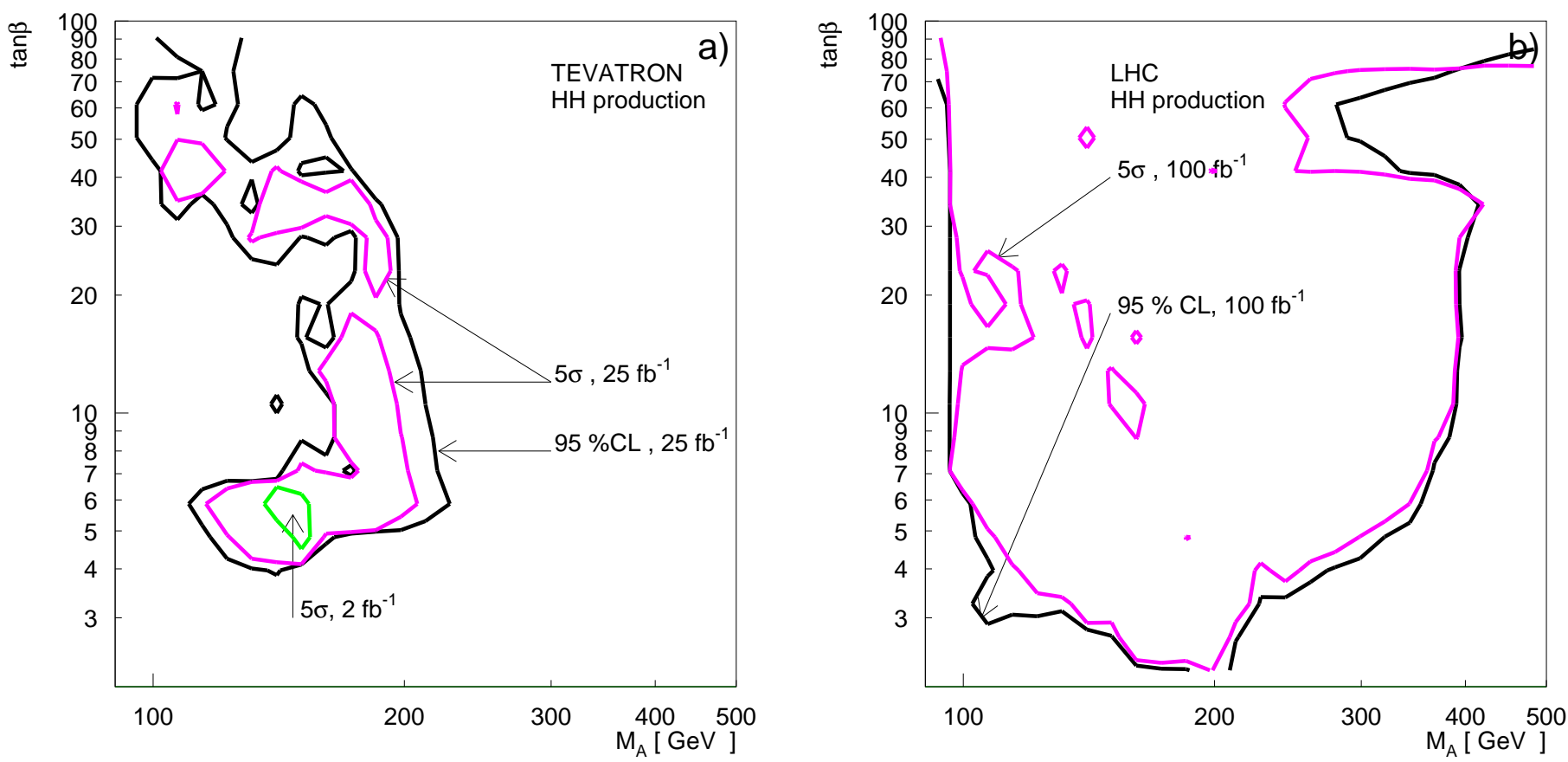

FIG. 7. 95\% c.l. exclusion and $5 \sigma$ discovery regions for heavy Higgs pair production at the Tevatron $\left(25 \mathrm{fb}^{-1}\right)(\mathrm{a})$ and LHC $\left(100 \mathrm{fb}^{-1}\right)(\mathrm{b})$, for "maximized" squark loop contributions (see the text for details). The light grey contour in (a) shows the region where a $\geq 5 \sigma$ signal should be detectable at the Tevatron with just $2 \mathrm{fb}^{-1}$ of data.

The answer is shown in Fig. 0, which shows the potential of the Tevatron and the LHC for observing a signal from $H H$ (heavy Higgs boson) pair production in the $4 b$ final state for the case of "maximal" squark loop contribution; as usual, contributions from nearly degenerate final states are included. We see that a significant part of the presently still allowed parameter space with $m_{A} \lesssim 200 \mathrm{GeV}$ can be probed with $25 \mathrm{fb}^{-1}$ of data at the Tevatron. LHC experiment will be able to extend this reach over most of the region where $m_{H} \leq 2 m_{t}$, if squark loop contributions are indeed very large. However, once $m_{H}>2 m_{t}$, the branching ratio of $H$ into $b \bar{b}$, and hence the signal in the $4 b$ final state, drops quickly. In principle one might then be able to extract a signal in the $4 t$ final state; however, since the decay of each top quark produces up to three separate jets, the kinematical reconstruction will be very difficult in this case. Similarly, for $m_{A}>200 \mathrm{GeV}, m_{H} \leq 2 m_{t}$ and small $\tan \beta$, $H \rightarrow h h$ decays dominate; $H H$ production would then lead to final states with up to 8 $b$-quarks! In this case it might be sufficient to simply require $\geq 5 b$-tags in the final state; kinematic reconstruction may not be necessary to suppress the background. 
It should be noted that the squark mass parameters used in Fig.7 usually differ from those used in Fig.5. Parts of the $\left(m_{A}, \tan \beta\right)$ plane that appear accessible in both the $h h$ and $\mathrm{HH}$ final states may therefore in fact not yield simultaneous signals in both channels. However, cases do exist where two distinct signals can be found, in particular at the LHC.

\section{SUMMARY AND CONCLUSIONS}

In this paper we have studied the potential of the Tevatron collider and the LHC for the search for the pair production of the neutral Higgs bosons of the MSSM in the $b \bar{b} b \bar{b}$ final state. To that end, we wrote Monte Carlo event generators for all signal and (irreducible) background processes. We included initial and final state showering, hadronization, and heavy hadron decay through an interface with PYTHIA/JETSET. We also introduced resolution smearing for jet energies.

We used these event generators to perform a detailed kinematical analysis with the aim of extracting the signal, working out the optimal set of kinematical cuts. The main outcome of this analysis are values of the minimal total signal cross section times branching ratio required for a $5 \sigma$ observation of the signal, as well as for placing $95 \%$ c.l. exclusion limits, at both the Tevatron and the LHC.

In the latter case we found that systematic errors play a crucial role. Due to the large event rate achievable at the LHC, the cross section required for a signal with $5 \sigma$ statistical significance corresponds to a signal to background ratio of just a few percent, for Higgs masses below $130 \mathrm{GeV}$. In our analysis we assigned a $2 \%$ systematic uncertainty (at the $1 \sigma$ level) to the background estimate. Clearly a detailed experimental analysis will be required to determine how (un)realistic this assumption is. Unfortunately, such an analysis may only be possible after LHC experimenters have had the opportunity to analyze real data on multi- $b$ final states.

We should emphasize here that our signal only includes the pair production of two neutral Higgs bosons through gluon fusion. Other contributions to the pair production of neutral Higgs bosons exist [29], but have far smaller cross sections. In contrast, the total cross section for associated Higgs $b \bar{b}$ production can be large at large $\tan \beta$ [30]; this also contributes to $4 b$ final states. However, in this case two of the $b$-jets are quite soft, and therefore have low tagging efficiencies. Most of the remaining contribution will be removed by our kinematical cuts. We therefore believe that our calculation includes the by far most important contribution to the signal.

Similarly, our background calculation only includes irreducible backgrounds from pure QCD $b \bar{b} b \bar{b}$ production, as well as from $Z b \bar{b}$ production followed by $Z \rightarrow b \bar{b}$ decay. We showed that "fake" backgrounds should be negligible if the "false positive" $b$-tag efficiency of light quark and gluon jets is a few percent or less. We also estimated backgrounds from multiparton $(4 \rightarrow 4)$ processes to be far smaller than those from $2 \rightarrow 4$ processes, and argued that the potentially sizable background from independent $p p$ interactions during the same bunch crossing at the LHC can be removed by requiring that all four $b$ jets come from the same primary vertex.

We did not attempt to estimate backgrounds from the production of supersymmetric particles. These frequently produce hard $b$-jets in their cascade decays [31], some of which might even come from the decay of on-shell Higgs bosons. However, the final state will 
then also contain additional energetic particles, in particular two LSP's. If these are stable or long-lived, the events will tend to have a large transverse momentum imbalance, unlike our signal. If the LSP's decay, the events will contain several hard photons, leptons and/or additional non $-b$ jets, and should thus again be easily distinguishable from the signal we are investigating. We therefore believe our background estimate to be reliable (with the caveats required for any leading order QCD calculation).

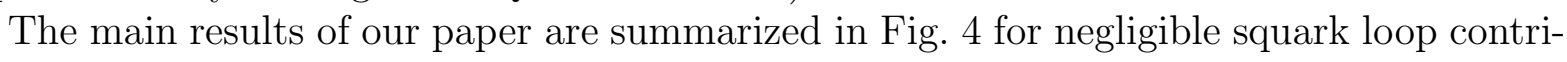
butions, and Figs. 5 and 7 for the case of very large squark loop contributions to the signal. The former case is not quite the most pessimistic one, since mild destructive interference between quark and squark loop contributions is possible [10]. However, we did not find any scenario where this reduces the cross section by more than $20 \%$ or so; this is hardly significant, given that our matrix elements are only calculated to lowest nontrivial order in QCD perturbation theory. Similarly, Figs. 5 and 7 probably do not quite show the most optimistic scenarios, since we had to use approximate search strategies of the squark parameter space when maximizing the signal.

In the absence of substantial squark loop contributions, the prospects for Tevatron experiments appear to be dim. Even with $25 \mathrm{fb}^{-1}$ of data only a tiny corner of the currently still allowed MSSM parameter space can be probed. Even LHC experiments can then only probe scenarios with $m_{A} \lesssim 300 \mathrm{GeV}$ and either very large or quite small values of $\tan \beta$.

On the other hand, if squark loop contributions are nearly maximal, and if it is possible to construct an efficient trigger for events containing $4 b$-jets with $\left\langle p_{T}\right\rangle \sim 50 \mathrm{GeV}$, LHC experiments should find a signal for $h$ production for practically all allowed combinations of $m_{A}$ and $\tan \beta$; HH production (augmented by nearly degenerate modes) should be visible for most scenarios with $m_{H} \leq 2 m_{t}$. Moreover, with $25 \mathrm{fb}^{-1}$ of data, Tevatron experiments would be sensitive to most of the region with $m_{A}<300 \mathrm{GeV}$; if $\tan \beta$ is large, even scenarios with $m_{A}>500 \mathrm{GeV}$ might be detectable. Our most exciting result is that a significant region of parameter space with $m_{A} \lesssim 250 \mathrm{GeV}$ should be accessible already at the next run of the Tevatron collider, which is projected to collect $2 \mathrm{fb}^{-1}$ of data. This seems to be the most robust signal for the production of MSSM Higgs bosons at the Tevatron that has been suggested so far.

However, these possibly very large squark loop contribution carry a price. First, it may not be easy to translate non-observation of a signal into a bound on MSSM parameter space. Not only the total cross section, but also kinematical distributions depend on the values of parameters describing both the Higgs sector and third generation squarks; even our simplified treatment ended up with five free parameters. Note that the kinematic characteristics of the signal depend not only on the masses of the Higgs bosons in the final state, but also on the masses of (s)particles in the dominant loop contributions to the signal, which determine the $\hat{s}$ dependence of the partonic cross section. Secondly, the presence of squark loop contributions of a priori unknown size might also make it difficult to use $h$-pair production to constrain the Higgs potential by measuring the Hhh coupling, as suggested in ref. [29]. On the other hand, the dependence of both the normalization and the shape of various differential signal cross sections on numerous MSSM parameters also means that a positive signal could teach us a great deal about both the Higgs and the squark sector of the theory.

In this article we have only considered the $4 b$ signal for Higgs boson pair production. Some 15 to $20 \%$ of all Higgs boson pairs will decay into $b \bar{b} \tau^{+} \tau^{-}$final states. While subdomi- 
nant, this mode will probably offer a better signal to background ratio after basic acceptance cuts. On the other hand, the presence of several neutrinos in the final state makes kinematic reconstruction more difficult. A dedicated signal and background analysis in this channel might nevertheless prove rewarding. Continuing in the direction of cleaner final states with smaller branching ratios, one might search for $b \bar{b} \gamma \gamma$ events. Even though the branching ratio is now only in the $10^{-3}$ range, an ATLAS study 28 found a better discovery reach for $H \rightarrow h h$ in this channel than in the $4 b$ channel. However, their study assumed that squarks are heavy; in general, squark loop contributions can reduce the branching ratios of Higgs bosons into two-photon final states [32]. We conclude that there is considerable room for further studies of the pair production of neutral Higgs bosons at hadron colliders.

\section{ACKNOWLEDGMENTS}

We thank Xerxes Tata and Pedro Mercadante for useful discussions, and Abdel Djouadi for a careful reading of the manuscript. M.D. thanks the theoretical particle physics group at the University of Hawaii at Honolulu as well as the school of physics of the Korea Institute of Advanced Studies, Seoul, for their hospitality. This work was supported by Fundação de Amparo à Pesquisa do Estado de São Paulo (FAPESP) and by U.S. Department of Energy under the contract DE-AC03-76SF00515. 


\section{REFERENCES}

[1] J. F. Gunion, A. Stange, and S. Willenbrock, hep-ph/9602238, in Electroweak Symmetry Breaking and New Physics at the TeV Scale, eds. T. L. Barklow, S. Dawson, H. E. Haber and J. L. Siegrist, World Scientific (1996); M. Carena, S. Mrenna and C. E. M. Wagner, hep-ph/9907422.

[2] A. Djouadi et al., hep-ph/9605437, in the Proceedings of the Workshop "Physics at Linear $e^{+} e^{-}$Colliders", Annecy-Gran Sasso-Hamburg, 1995.

[3] A. Stange, W. Marciano and S. Willenbrock, Phys. Rev. D49, 1354 (1994);

A. Belyaev, E. Boos, and L. Dudko, Mod. Phys. Lett. A10, 25 (1995).

[4] R. Kleiss, Z. Kunszt and W. J. Stirling, Phys. Lett. B253, 269 (1991).

[5] J. F. Gunion, Phys. Lett. B261, 510 (1991).

[6] O. J. P. Éboli, G. C. Marques, S. F. Novaes, and A. A. Natale, Phys. Lett. B197, 269 (1987); D. A. Dicus, C. Kao and S. Willenbrock, Phys. Lett. B203, 457 (1988); E. W. N. Glover and J. J. van der Bij, Nucl. Phys. B309, 282 (1988).

[7] For a review, see H. E. Haber and G. L. Kane, Phys. Rep. 117, 75 (1985).

[8] J. Dai, J. F. Gunion and R. Vega, Phys. Lett. B371, 71 (1996), and Phys. Lett. B387, 801 (1996).

[9] T. Plehn, M. Spira, and P. M. Zerwas, Nucl. Phys. B479, 46 (1996); Erratum: ibid. B531, 655 (1998).

[10] A. Belyaev, M. Drees, O. J. P. Éboli, J. K. Mizukoshi and S. F. Novaes, Phys. Rev. D60, 075008 (1999).

[11] T. Sjöstrand, Comp. Phys. Comm. 82, 74 (1994).

[12] E. E. Boos et al., hep-ph/9503280, SNUTP-94-116; P. Baikov et al., in Proc. of the Xth Int. Workshop on High Energy Physics and Quantum Field Theory, QFTHEP-95, eds. B. Levtchenko and V. Savrin, (Moscow, 1995), p.101.

[13] CTEQ collab., H. L. Lai et al., Phys. Rev. D55, 1280 (1997), hep-ph/9606399.

[14] A. Djouadi, M. Spira, and P. M. Zerwas, Phys. Lett. B264, 440 (1991); S. Dawson, Nucl. Phys. B359, 283 (1991); M. Spira, A. Djouadi, D. Graudenz, and P. M. Zerwas, Nucl. Phys. B453, 17 (1995).

[15] Y. Okada, M. Yamaguchi and T. Yanagida, Prog. Theor. Phys. 85, 1 (1991); H.E. Haber and R. Hempfling, Phys. Rev. Lett. 66, 1815 (1991).

[16] J. Ellis, G. Ridolfi and F. Zwirner, Phys. Lett. B262, 477 (1991).

[17] Proc. of Supersymmetry/Higgs RUN II workshop, February - November, 1998, http://fnth37.fnal.gov/susy.htm

[18] F. Pauss and M. Dittmar, report ETHZ-IPP-PR-98-09, hep-ex/9901018.

[19] M. Drees and M. M. Nojiri, Phys. Rev. D45, 2482 (1992); H. E. Haber, R. Hempfling and A. Hoang, Z. Phys. C75, 539 (1997), hep-ph/9609331.

[20] The LEP collaborations (ALEPH, DELPHI, L3 and OPAL), CERN-EP-99-060; OPAL collab., G. Abbiendi et al, hep-ex/9908002.

[21] See e.g. J. F. Gunion, H. E. Haber, G. L. Kane, and S. Dawson, The Higgs Hunter's Guide, Addison-Wesley (1990).

[22] OPAL collab., G. Abbiendi et al., Phys. Lett. B456, 95 (1999), hep-ex/9903070.

[23] M. Drees and K. Hagiwara, Phys. Rev. D42, 1709 (1990).

[24] J. Erler and P. Langacker, hep-ph/9809352, to appear in the proceedings of WEIN 98, Santa Fe, NM, June 1998. 
[25] J. M. Frere, D. R. T. Jones and S. Raby, Nucl. Phys. B222, 11 (1983).

[26] CDF collab., F. Abe et al., Phys. Rev. D56, 3811 (1997).

[27] CMS collab., S. Abdullin et al., CMS-NOTE-1998-006, hep-ph/9806366

[28] See Chapter 19 of the ATLAS Technical Design Report, http://atlasinfo.cern.ch/Atlas/GROUPS/PHYSICS/TDR/access.html.

[29] A. Djouadi, W. Kilian, M. Muhlleitner and P. M. Zerwas, DESY 99/033, hepph/9904287.

[30] J. Lorenzo Diaz-Cruz, Hong-Jian He, T. Tait, and C. P. Yuan, Phys. Rev. Lett. 80, 4641 (1998); C. Balazs, J. L. Diaz-Cruz, H. J. He, T. Tait, and C. P. Yuan, Phys. Rev. D59, 055016 (1999).

[31] See e.g. H. Baer, C.-H. Chen, M. Drees, F. Paige and X. Tata, Phys. Rev. D59, 055014 (1999), hep-ph/9809223, and references therein.

[32] A. Djouadi, Phys. Lett. B435, 101 (1998), hep-ph/9806315. 